\title{
Cohort study examining tamoxifen adherence and its relationship to mortality in women with breast cancer
}

\author{
C McCowan*,', J Shearer², PT Donnan', JA Dewar², M Crilly ${ }^{3}$, AM Thompson ${ }^{4}$ and TP Fahey ${ }^{1,5}$ \\ 'Division of Community Health Sciences, University of Dundee, MacKenzie Building, Kirsty Semple Way, Dundee DD2 4BF, UK; ${ }^{2}$ Department of \\ Radiotherapy and Oncology, Ninewells Hospital and Medical School, Dundee DDI 9SY, UK; ${ }^{3}$ Department of Public Health, University of Aberdeen, \\ School of Medicine, Polwarth Building, Aberdeen AB25 2ZD, UK; ${ }^{4}$ Department of Surgery and Molecular Oncology, University of Dundee, Ninewells \\ Hospital and Medical School, Dundee DDI 9SY, UK; ${ }^{5}$ Department of General Practice, Royal College of Surgeons in Ireland, 120 St Stephens Green, \\ Dublin 2, Ireland
}

Increasing duration of tamoxifen therapy improves survival in women with breast cancer but the impact of adherence to tamoxifen on mortality is unclear. This study investigated whether women prescribed tamoxifen after surgery for breast cancer adhered to their prescription and whether adherence influenced survival. A retrospective cohort study of all women with incident breast cancer in the Tayside region of Scotland between 1993 and 2002 was linked to encashed prescription records to calculate adherence to tamoxifen. Survival analysis was used to determine the effect of adherence on all-cause mortality. In all 2080 patients formed the study cohort with 1633 (79\%) prescribed tamoxifen. The median duration of use was 2.42 years (IQR $=1.04-4.89$ years). Longer duration was associated with better survival but this varied over time. The hazard ratio for mortality in relation to duration at 2.4 years was $0.85,95 \% \mathrm{Cl}=0.83-0.87$. Median adherence to tamoxifen was $93 \%$ (interquartile range $=84-100 \%$ ). Adherence $<80 \%$ was associated with poorer survival, hazard ratio I. I0,95\% Cl=1.00 I- I.2I. Persistence with tamoxifen was modest with only $49 \%$ continuing therapy for 5 years of those followed up for 5 years or more. Increased duration of tamoxifen reduces the risk of death, although one in two women do not complete the recommended 5-year course of treatment. A significant proportion of women have low adherence to tamoxifen and are at increased risk of death.

British Journal of Cancer (2008) 99, I763- 1768. doi:I0.1038/sj.bjc.6604758 www.bjcancer.com

Published online 4 November 2008

(c) 2008 Cancer Research UK

Keywords: breast cancer; tamoxifen; adherence; mortality; community

Tamoxifen is an important adjuvant therapy for patients with breast cancer (Osborne, 1998) and reduces the breast cancer mortality rate over 15 years by $31 \%$ in women with oestrogen receptor-positive early breast cancers. (Early Breast Cancer Trialists' Collaborative, 2005) Tamoxifen (20 mg once daily) is recommended for patients for 5 years adjuvant therapy (Early Breast Cancer Trialists' Collaborative Group, 2004) to provide maximum benefit (Fisher et al, 1996; Stewart et al, 1996; Burstein and Winer, 2000).

Adherence to a prescribed medication can be defined as: 'the extent to which a patient's behaviour ... coincides with medical or health advice' (McDonald et al, 2002). Non-adherence to prescribed medication for patients with chronic conditions is a major public health issue. The level of non-adherence reported by studies is large, up to $50 \%$, and many studies have shown worse clinical outcomes associated with low adherence (DiMatteo, 2004).

\footnotetext{
*Correspondence: C McCowan, MRC Research Training Fellow, Division of Community Health Sciences, University of Dundee, MacKenzie Building, Kirsty Semple Way, Dundee DD2 4BF, UK.

E-mail: c.mccowan@chs.dundee.ac.uk

Received I July 2008; revised 24 September 2008; accepted I October 2008; published online 4 November 2008
}

The literature for tamoxifen deals with three different aspects of adherence to medication. The first, duration of tamoxifen therapy has an effect on disease recurrence and mortality from breast cancer with 5 years being the recommended treatment period (Swedish Breast Cancer Cooperative Group, 1996; Fisher et al, 2001; Yood et al, 2008). A second closely related aspect of adherence is persistence or continuation of therapy where the period that a patient continues to take the medication before a prespecified break in medication is measured. This differs from duration in that if a patient restarts therapy after a break of the specified length this additional use of medication is ignored. Several recent studies have reported non-persistence with tamoxifen therapy. The first reported cumulative non-persistence for tamoxifen in a cohort of 2816 women at $22.1 \%$ after 1 year and $35.2 \%$ at 3.5 years (Barron et al, 2007). The second followed 961 women over 5 years and found that $49 \%$ stopped taking their tamoxifen before the recommended 5-year treatment period (Owusu et al, 2008). An earlier study reported $17 \%$ of women aged $65+$ years had stopped treatment within 2 years (Fink et al, 2004). However, none of these studies assessed the impact of failure to persist with therapy on mortality or the independent effect of non-adherence in patients taking tamoxifen.

The last aspect of adherence to tamoxifen is whether the patients take the medication consistently on a daily basis as prescribed. A recent review concluded that this aspect of non-adherence to 
adjuvant medication may limit the efficacy of breast cancer treatment, although there was limited evidence for this (Chlebowski et al, 2006). Adherence to adjuvant tamoxifen during clinical trials is high, with reported levels of $94-100 \%$ (Fisher et al, 1994, 1996; Dewar et al, 1996). Community-based studies found that $23 \%$ of patients failed to take tamoxifen in 1 out of 5 days with adherence falling from 89 to $50 \%$ over 4 years (Partridge et al, 2003) and that deviations from prescribed tamoxifen therapy were common (Demissie et al, 2001). A small survey on self reported adherence in 53 women found that $62 \%$ acknowledged having missed at least one dose of tamoxifen in the previous 6 months, with $24 \%$ missing at least one dose per week (Murthy et al, 2002). These studies suggest that non-adherence to tamoxifen is underestimated and further understanding of the relationship to mortality is required. No previous study has examined the overall effect on all-cause mortality of duration of use, adherence to and persistence with tamoxifen either in the hospital or community setting.

The aim of this study was to investigate adherence to tamoxifen for all women with breast cancer drawn from a defined geographical population in Tayside, Scotland. The objectives were to:

Describe the duration of use, adherence to and persistence with tamoxifen in a geographically defined population of women.

Investigate whether duration and adherence to tamoxifen influenced all-cause mortality after adjusting for important clinical and demographic factors.

\section{MATERIALS AND METHODS}

\section{Identification of study cohort}

Women resident in Tayside registered with the Cancer Registry as having breast cancer or with an initial hospital admission for breast cancer from 1993 to January 2002 were identified. Only those women who were resident for the entire period of the study or until death were included. Patients who had prescribing or hospital admission records, an audit record and a Cancer Registry record within the study period comprised the population of interest. Every patient registered with a general practitioner in Tayside is assigned a 10 digit unique patient identifier, the Community Health Index number (CHI number) used in all NHS encounters. The CHI number, which includes the patient's date of birth, allows linkage of health-related datasets providing a unique resource combining information on dispensed prescribing with detailed clinical data at the individual patient level.

\section{Study covariates}

Date of diagnosis was recorded from either the Cancer Registry or the clinical audit records and age at diagnosis was then calculated. Duration of breast cancer was calculated from the date of diagnosis to the date of death or until 7 January 2002, which was the study cutoff point. Each patient had a Carstairs score (a measure of deprivation (Carstairs and Morris, 1990)) and subsequent category calculated based on their home postcode using census data from 2001. There were only two patients in Carstairs category 7 , the most deprived grouping, and so categories 6 and 7 were grouped together for analysis.

A Charlson's co-morbidity index, which included a category for presence of cancer, was derived for each patient using standard procedures and the ICD codes version 9 (Deyo et al, 1992) and 10 (Sundararajan et al, 2004) in the hospital admission records. The encashed prescribing records were also examined and the Charlson disease groups were flagged for patients who were receiving medication for respiratory disease, AIDS, peptic ulcers, cancer, metastatic tumours, diabetes, myocardial infarction, connective tissue disorders and dementia. Charlson Index scores were calculated and categorised into three groups with low $(0-2)$, medium (3-5) and high morbidity $(6+)$. The number of different preparations prescribed to patients' for the 6 months prior to diagnosis was counted and categorised into four groups, $0,1-2$, $3-5$ or $6+$. The characteristics of the tumour on presentation were recorded using clinical TNM categories (tumour size, presence of regional node metastasis and distant metastases), pathological grading, pathological description of axillary node metastasis and oestrogen receptor status. Where conflicts existed between the cancer registry and clinical audit records, the audit records were used as the primary source of information.

\section{Tamoxifen adherence}

Each prescription for tamoxifen was analysed and the number of days covered by that prescription recorded from the number of dispensed tablets and daily dose. The duration of tamoxifen therapy was calculated from the number of days between the first and last prescription and the coverage of the last prescription. The adherence index for each patient was calculated by: summing the coverage for all the prescriptions for each patient, dividing it by the duration, and then converting this to a percentage (see Figure 1). The adherence index was calculated across the entire duration of therapy whether this exceeded 5 years or not. Based on the existing literature (Murthy et al, 2002; Wei et al, 2002; Partridge et al, 2003) patients with an adherence index less than $80 \%$ were deemed to have 'low adherence' (Osterberg and Blaschke, 2005).

To allow a direct comparison with previous studies, (Barron et al, 2007) persistence was calculated as the length of time from first prescription to a break of at least 180 days before the completion of 5 years of therapy. Patients who continued treatment had their length of persistence calculated using either the date of death or the end of the study period.

\section{Outcome variable}

The main outcome measure was all-cause mortality recorded on the General Registry Office death certificate.

\section{Statistical analysis}

Data were described as number of subjects (percentages) for categorical variables and mean (s.d.) for continuous variables. Where continuous variables did not follow a normal distribution, tested using the Shapiro-Wilks test for skewness, the median and interquartile range were reported. $\chi^{2}$ tests for trend ( $\chi^{2}$ trend, d.f., $P$ ) were reported for differences in distribution of the population with $n$ categories. Cox Proportional Hazards models were utilised to estimate hazard ratios and $95 \%$ confidence intervals for each unadjusted and adjusted covariate for all-cause mortality. Patients were followed up from date of diagnosis or commencement of tamoxifen until time of death or the end of the study.

\section{- Individual patient has encashed the prescriptions below}

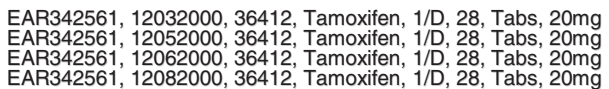

EAR342561, 12062000, 36412, Tamoxifen, 1/D, 28, Tabs, $20 \mathrm{mg}$

- No of days coverage $=4 \times 28=112$

- Calculate duration of therapy from first and last prescription

- Duration is from $12 / 3 / 2000$ to $12 / 08 / 2000+28$ days $=182$

- Adherence index $=(112 / 182) \times 100=62 \%$

Figure I Calculation of adherence and duration. 
Multivariable results are presented as hazard ratios and $95 \%$ confidence intervals. Covariates were included in the multivariate model if they were deemed to be of clinical significance or had univariable $P$-value $<0.2$. The proportional hazards assumption was assessed using trend-tests of the Schoenfeld residuals and those which failed the assumption or which were deemed to be time-dependent were entered as continuous time-dependent covariates (Bradburn et al, 2003).

In a community-based, non-randomised study many known and unknown factors may determine who receives tamoxifen, which can potentially bias results. A propensity score estimating the probability of a patient receiving tamoxifen was calculated using a logistic regression model for age, social class, pathological tumour characteristics, Charlson's index and co-prescribing (Wang and Donnan, 2001). This was added to the model to adjust for propensity to receive tamoxifen and so reduce bias in the survival analysis. All statistical analyses were performed using Stata version 9.

The study was granted approval from the Tayside Committee on Medical Research Ethics and the Caldicott Guardian.

\section{RESULTS}

A total of 2184 patients were identified with prescribing, hospital admission records for breast cancer surgery, cancer audit and cancer registry records for the period of the study. Patients who were known to have had cancer before 1993, or who received tamoxifen more than 6 months prior to their diagnosis or those diagnosed with ductal carcinoma in situ alone were excluded. This left a total of 2080 patients whose records were analysed within this study. The mean age at diagnosis was 61.4 (s.d. $=14.02$ ) and patients were followed up for a total of 7619 person years with a median follow-up of 3.16 years (interquartile range $=1.38-5.72$ years). A total of 511 deaths (25\%) were recorded.

\section{Descriptive statistics for duration, adherence and persistence with tamoxifen}

Tamoxifen was prescribed as therapy to 1633 (79\%) patients of whom $414(25 \%)$ died during the study. The median duration of tamoxifen in the study was 2.42 years (interquartile range $=1.04$ 4.89 years). Patients were generally highly adherent to their medication during the course of treatment with a median adherence of $93 \%$ (interquartile range $=84-100 \%$ ). There were $315(19 \%)$ patients with low adherence of less than $80 \%$. There was no difference in low adherence by social class $(P=0.96)$ but there was a trend for a higher proportion of younger women to have low adherence $(P<0.001)$.

However, 411 (33\%) patients prescribed tamoxifen discontinued their medication before completing 5 years of treatment. Within 1 year of commencing tamoxifen $10 \%$ of patients had discontinued treatment, for patients followed for 2 years or more $19 \%$ patients had discontinued treatment, $32 \%$ patients for those followed for three and a half years or more and $51 \%$ patients of those followed for 5 years plus.

\section{Comparison of tamoxifen users and non users}

The distribution of age, social class, Charlson's index and co-prescribing is presented in Table 1 . Women in the younger age groups were less likely to receive tamoxifen than older patients $(P<0.001)$; social class categories 2 and 3 had the highest proportion of women not receiving the drug $(P=0.011)$ and patients who received tamoxifen had higher levels of co-prescribing $(P<0.001)$ and greater Charlson's Index scores $(P<0.001)$.
Table I Descriptive statistics of cohort- age, social class, Charlson's index and co-prescribing

\begin{tabular}{|c|c|c|c|}
\hline & $\begin{array}{l}\text { Patients with } \\
\text { tamoxifen (\%) }\end{array}$ & $\begin{array}{c}\text { No tamoxifen } \\
(\%)\end{array}$ & $\begin{array}{l}\text { Wald } \chi^{2} \text { test } \\
\text { (Wald, d.f., } P \text { ) }\end{array}$ \\
\hline Numbers & $1633(79)$ & $447(21)$ & \\
\hline \multicolumn{4}{|l|}{ Age group } \\
\hline$<40$ & $84(5)$ & $37(8)$ & $22.6,5, P<0.001$ \\
\hline $40-49$ & $237(15)$ & $94(21)$ & \\
\hline $50-59$ & 435 (27) & $112(25)$ & \\
\hline $60-69$ & $365(22)$ & $95(2 \mathrm{l})$ & \\
\hline $70-79$ & $331(20)$ & $77(17)$ & \\
\hline $80+$ & $181(11)$ & $32(7)$ & \\
\hline \multicolumn{4}{|c|}{ Carstairs category } \\
\hline I & $152(9)$ & $29(7)$ & $|4.9,5, P=0.01|$ \\
\hline 2 & 327 (20) & $108(24)$ & \\
\hline 3 & $443(27)$ & 145 (32) & \\
\hline 4 & 306 (19) & $66(15)$ & \\
\hline 5 & $164(10)$ & $34(8)$ & \\
\hline $6 / 7$ & $237(15)$ & $64(14)$ & \\
\hline \multicolumn{4}{|c|}{ Number of prescriptions at diagnosis } \\
\hline 0 & $500(31)$ & $254(57)$ & $104.9,3, P<0.00 \mid$ \\
\hline $1-2$ & 457 (28) & 77 (17) & \\
\hline $3-5$ & $397(24)$ & $63(14)$ & \\
\hline $6+$ & $279(17)$ & $53(12)$ & \\
\hline \multicolumn{4}{|c|}{ Charlson's index } \\
\hline $0-2$ & 476 (29) & $198(44)$ & $37.9,2, P<0.001$ \\
\hline $3-5$ & $510(31)$ & $100(22)$ & \\
\hline $6+$ & 647 (40) & 149 (33) & \\
\hline
\end{tabular}

\section{Patient characteristics and tamoxifen usage}

The clinical and pathological characteristics of the cancer at diagnosis are shown in Table 2. There were no differences in clinical tumour stage $(P=0.86)$ between tamoxifen users and those who did not receive the drug. However, patients were less likely to receive tamoxifen therapy if they had positive clinical nodes at initial diagnosis $(P=0.001)$, if metastases were present $(P=0.03)$; if tumour grade was worse $(P<0.001)$, if patients had positive nodes on pathology $(P<0.001)$ or if they had negative oestrogen receptor status $(P<0.001)$.

\section{Cox proportional hazards model for all-cause mortality}

An initial multivariate model was created to investigate the effect of tamoxifen use and showed that patients not prescribed tamoxifen were at significantly higher risk of death than patients who were prescribed tamoxifen after allowing for all other covariates $(\mathrm{HR}=1.36,95 \% \mathrm{CI}=1.05-1.76)$.

A subsequent multivariate model investigated the adjusted effect of covariates for those patients who used tamoxifen. Adjusting for all factors, increasing age, increasing tumour grade, positive or unknown pathological node status and negative or unknown oestrogen receptor status increased the risk of death. A Charlson's Index score of 6 or more also increased the risk of death over time, at the median duration of 2.4 years, the hazard ratio increased by $1.59,95 \% \mathrm{CI}=1.14-2.21$. Duration of tamoxifen use was also associated with better survival after allowing for other covariates and the hazard ratio at a given time could be calculated using the equation $\mathrm{HR}=\exp (-0.065 \times$ follow-up time $)$. At 2.4 years, the median duration, the hazard ratio was $0.85,95 \% \mathrm{CI}=0.83-0.87$ (see Table 3).

Patients with an adherence index of $<80 \%$ had an increased hazard of death calculated using the equation $\mathrm{HR}=\exp (0.04 \times$ 
Table 2 Clinical and pathological characteristics of cancer at initial diagnosis

\begin{tabular}{|c|c|c|c|}
\hline & $\begin{array}{l}\text { Patients with tamoxifen } \\
\text { (\%) }\end{array}$ & $\begin{array}{c}\text { No tamoxifen } \\
(\%)\end{array}$ & $\begin{array}{l}\text { Wald } \chi^{2} \text { test } \\
\text { (Wald, d.f., } P \text { ) }\end{array}$ \\
\hline Numbers & $1633(79)$ & $447(21)$ & \\
\hline \multicolumn{4}{|l|}{ Tumour stage } \\
\hline 1 & $440(27)$ & $119(27)$ & $1.3,4, P=0.86$ \\
\hline 2 & $522(32)$ & $136(30)$ & \\
\hline 3 & $118(7)$ & $36(8)$ & \\
\hline 4 & $|4|(9)$ & $35(8)$ & \\
\hline Unknown & $412(25)$ & $121(27)$ & \\
\hline \multicolumn{4}{|l|}{ Clinical nodes } \\
\hline 0 & $1144(70)$ & $316(7 \mid)$ & $17.7,3, P=0.00 \mid$ \\
\hline । & $230(14)$ & $74(17)$ & \\
\hline 2 & $34(2)$ & $20(4)$ & \\
\hline Unknown & $225(14)$ & $37(8)$ & \\
\hline \multicolumn{4}{|c|}{ Clinical metastases } \\
\hline No & I354 (83) & $365(82)$ & 7.I, $2, P=0.03$ \\
\hline Yes & $72(4)$ & $33(7)$ & \\
\hline Unknown & $207(13)$ & $49(\mid 1)$ & \\
\hline \multicolumn{4}{|l|}{ Tumour grade } \\
\hline 1 & $221(14)$ & $48(11)$ & $86.7,3, P<0.001$ \\
\hline 2 & $545(33)$ & $128(29)$ & \\
\hline 3 & $363(22)$ & $194(43)$ & \\
\hline Unknown & $504(31)$ & $77(17)$ & \\
\hline \multicolumn{4}{|c|}{ Oestrogen receptor status } \\
\hline Positive & $869(53)$ & $142(32)$ & $\begin{array}{l}227.1,2 \\
P<0.001\end{array}$ \\
\hline Negative & $199(12)$ & $195(43)$ & \\
\hline Unknown & $565(35)$ & $110(25)$ & \\
\hline \multicolumn{4}{|c|}{ Pathological nodes } \\
\hline Negative & 754 (46) & $230(52)$ & $|6.4,2, P<0.00|$ \\
\hline Positive & $446(27)$ & $140(31)$ & \\
\hline Unknown & $433(27)$ & $77(17)$ & \\
\hline
\end{tabular}

follow-up time). At the median duration of tamoxifen use of 2.4 years the hazard ratio for low adherence was $1.10,95 \% \mathrm{CI}=1.001-$ 1.21 (Table 3).

Tamoxifen is not recommended for use in patients with oestrogen receptor negative tumours, although within our study population it was used with this patient group. Restricting the analysis to patients with ER + ve or ER status unknown was associated with a significantly increased hazard of death for adherence below $80 \%, \mathrm{HR}=\exp (0.05 \times$ follow-up time $), 95 \% \mathrm{CI}$ $0.01-0.10$, the hazard ratio at the median duration being $1.13,95 \%$ $\mathrm{CI}=1.01-1.26$.

\section{DISCUSSION}

This study suggests that adherence to taking daily tamoxifen in the community setting is high, over four-fifths of women prescribed tamoxifen had an adherence of $80 \%$ or above during the course of treatment. However, cumulative persistence with tamoxifen treatment is low, with $51 \%$ of the patient population followed for 5 years or more discontinuing treatment by 5 years. The study defined high adherence at $80 \%$ or over and there was a significant increase in hazard of death for patients with adherence beneath that level. This suggests that the longer a patient has low adherence the greater their increase in hazard of death. Patients who were prescribed tamoxifen had better survival after adjusting for other
Table 3 Multivariate association between covariates and all cause mortality

\begin{tabular}{|c|c|c|c|}
\hline \multirow[b]{2}{*}{ Predictor } & \multicolumn{3}{|c|}{ Adjusted for other covariates } \\
\hline & HR & $95 \% \mathrm{Cl}$ & \\
\hline Age ( 10 years) & 1.15 & $1.05-1.27$ & $P=0.004$ \\
\hline \multicolumn{4}{|l|}{ Carstairs category } \\
\hline I (most affluent) & 1.0 & & \\
\hline 2 & 0.86 & $0.56-1.32$ & $P=0.486$ \\
\hline 3 & 1.03 & $0.69-1.54$ & $P=0.878$ \\
\hline 4 & 1.23 & $0.81-1.86$ & $P=0.333$ \\
\hline 5 & 1.04 & $0.66-1.65$ & $P=0.851$ \\
\hline 6/7 (most deprived) & 1.27 & $0.82-1.97$ & $P=0.353$ \\
\hline \multicolumn{4}{|l|}{ Charlson's index (at 2.4 years) } \\
\hline $0-2$ & 1.0 & & \\
\hline $3-5$ & 1.21 & $0.83-1.78$ & $P=0.325$ \\
\hline $6+$ & 1.59 & $1.14-2.21$ & $P=0.006$ \\
\hline \multicolumn{4}{|l|}{ Number of prescriptions at diagnosis } \\
\hline 0 & 1.0 & & \\
\hline $1-2$ & 0.75 & $0.46-1.23$ & $P=0.253$ \\
\hline $3-5$ & 0.56 & $0.22-1.22$ & $P=0.143$ \\
\hline $6+$ & 0.44 & $0.16-1.18$ & $P=0.104$ \\
\hline \multicolumn{4}{|l|}{ Tumour grade } \\
\hline \multicolumn{4}{|l|}{ I } \\
\hline 2 & 2.49 & $1.24-4.98$ & $P=0.010$ \\
\hline 3 & 4.03 & $2.01-8.08$ & $P<0.001$ \\
\hline Unknown & 2.22 & $0.89-5.54$ & $P=0.089$ \\
\hline \multicolumn{4}{|l|}{ Oestrogen receptor status } \\
\hline Positive & 1.0 & & \\
\hline Negative & 1.96 & $1.46-2.62$ & $P<0.001$ \\
\hline Unknown & 1.47 & $1.10-1.97$ & $P=0.009$ \\
\hline \multicolumn{4}{|l|}{ Pathological nodes } \\
\hline Negative & 1.0 & & \\
\hline Positive & 2.05 & $1.54-2.72$ & $P<0.001$ \\
\hline Unknown & 2.19 & $1.57-3.04$ & $P<0.001$ \\
\hline Propensity score & 0.01 & $0.00-3.30$ & $P=0.106$ \\
\hline Duration of tamoxifen (at 2.4 years) & 0.85 & $0.83-0.87$ & $P<0.001$ \\
\hline Adherence $<80 \%$ (at 2.4 years) & 1.10 & $1.001-1.21$ & $P=0.046$ \\
\hline
\end{tabular}

factors than those who did not, and the duration of tamoxifen use was also seen to reduce the hazard of death.

This study supports the use of tamoxifen in the community setting as an adjuvant therapy that reduces mortality in women with breast cancer. Though the reduction in hazard in this community-based study is lower, the higher survival figures reported in randomised controlled trials may reflect different inclusion criteria or some selection bias in terms of recruiting 'healthier' patients with less co-morbid illness to these trials.

In terms of generalisability, the study population comprised of women who had an initial attendance at a hospital for breast cancer, had an accompanying cancer registry record entry and a history of prescribing. The women also needed to be resident in the region served by the breast cancer service for the entire study period or until death to ensure that there was no loss to follow-up. Thus the study cohort of 2184 women accounts for $85 \%$ of potential patients.

Around three-quarters of breast cancer patients in Tayside received tamoxifen as adjuvant therapy, as reported elsewhere (Demissie et al, 2001). Approximately one-fifth of tamoxifen users had adherence below $80 \%$ similar to studies elsewhere (Murthy et al, 2002; Partridge et al, 2003). Non-persistence with tamoxifen therapy at 1 year differed from the figure reported in the study by Barron et al (2007) but at three and a half years was at a similar 
level and was similar to the figure reported by Fink et al (2004) at 2 years and Owusu et al (2008) at 5 years. Hence, the characteristics of tamoxifen use, adherence and persistence in this population is similar to those from other published work. This study concentrated on the duration of tamoxifen use and adherence to taking it on a prescribed daily basis, as this is a more complete marker of use than non-persistence. What is added to the existing literature is the linkage between low adherence and increased all-cause mortality.

The use of the propensity score within the final model helps reduce potential selection bias (Wang and Donnan, 2001) because of the use of observational data, where clinicians may choose patients with a lower risk of mortality to receive tamoxifen. However, our analysis holds after allowing for the predisposition of a patient to receive tamoxifen. With $79 \%$ of patients receiving tamoxifen it is unlikely that they were selected as having better survival prospects.

In terms of potential shortcomings, this study measured the adherence to medication by looking at the encashment of prescriptions. Although there are advantages and disadvantages to various techniques for measuring adherence, (Osterberg and Blaschke, 2005) and whereas we cannot tell if patients actually took the medication, breast cancer patients are probably motivated to be adherent, as reflected in the high levels of adherence reported by this and other studies. Tayside has a closed pharmacy system as all encashed prescriptions are returned to a central government office for reimbursement and this allowed us to measure adherence objectively across the whole population.

There was approximately 1 in 5 patients with less than $80 \%$ adherence, which means this study had low power to determine if adherence has an effect on mortality. In addition, it is now appreciated that ER-negative patients (200 in this study) do not benefit from adjuvant tamoxifen and would also reduce power. The study aimed to look at the effect of adherence to tamoxifen on survival in a broad range of women with breast cancer-prescribed tamoxifen in the community. Consequently, we included patients in the study cohort who would not have been eligible for inclusion in trials or where current treatment guidelines would not recommend its use. There were 105 patients with clinical metastases who received tamoxifen and such women may have had a different motivation to take their medication than those receiving it as adjuvant therapy. As the aim of our study was to assess the effect of adherence to tamoxifen in the community, using a comprehensive prescribing dataset to monitor long-term adherence, we intentionally included both patients with clinical metastases and also patients who were ER negative in our analysis. The effect of adherence to tamoxifen on survival was still evident in subsequent analysis performed on the cohort with these patient groups excluded.

This study could not explain why some women were not adherent to or discontinued their medication, although it suggested younger women were more likely to have low adherence. Other work agrees with this finding and also suggests side effects or a perception of low benefits from taking medication leads to

\section{REFERENCES}

Barron TI, Connolly R, Bennett K, Feely J, Kennedy MJ (2007) Early discontinuation of tamoxifen. In Cancer 109: 832-839

Bradburn MJ, Clark TG, Love SB, Altman DG (2003) Survival analysis Part III: multivariate data analysis - choosing a model and assessing its adequacy and fit. $\mathrm{Br} J$ Cancer 89: 605-611

Burstein HJ, Winer EP (2000) Primary care for survivors of breast cancer. New Engl J Med 343: 1086 - 1094

Carstairs V, Morris R (1990) Deprivation and health in Scotland. Health Bull 48: $162-175$

Chlebowski RT, Geller ML, Chlebowski RT, Geller ML (2006) Adherence to endocrine therapy for breast cancer. Oncology 71: 1-9 lower adherence in younger women (Fink et al, 2004; Grunfeld et al, 2005; Chlebowski et al, 2006).

The study did not report on chemotherapy or radiotherapy use. Treatment in Tayside used standard practises for both treatments over the study period so it seems unlikely that there could be any systematic difference in chemotherapy or radiotherapy use between any of the groups of interest. Furthermore, radiotherapy affects mortality only after $10-15$ years so it would not have been a factor within our analysis (Clarke et al, 2005).

Recurrence of breast cancer was also omitted because of the difficulties in ascertaining recurrence within a community setting. The routine datasets used do not record cancer recurrences directly and so a proxy measure would have been required which we decided against. Although this would not affect the main outcome of mortality we may have overestimated the number of women who discontinue their medication.

Lastly, the outcome used within the study was all-cause mortality selected as the accurate recording of cause of death has been questioned in the literature (Clark et al, 2003).

This study confirms that around $20 \%$ of patients have an adherence level, $<80 \%$, the equivalent of missing at least one tablet every 5 days. Our findings suggest that adherence to tamoxifen beneath this level has a negative effect on survival. The relatively long half-life of tamoxifen (Osborne, 1998) may mean that missing an occasional tablet is less of an issue and that the patients will still benefit from tamoxifen therapy. Aromatase inhibitors are increasingly used as adjuvant therapy but have a shorter half-life than tamoxifen and so strict adherence to this medication regimen should be emphasised as the occasional missed dose may have a greater detrimental effect on survival.

\section{CONCLUSION}

Patients who are prescribed adjuvant tamoxifen in the community have a lower risk of death with increased duration of use further reducing the risk. Cumulative non-persistence with tamoxifen therapy occurs in nearly half of patients prescribed tamoxifen. Conversely, in those who continue to take tamoxifen adherence is generally high, but there is a significant proportion of women with low adherence who are at greater risk of death. Patients need to be encouraged to continue their medication for the full 5 -year recommended period to ensure their best chance of survival.

\section{ACKNOWLEDGEMENTS}

We thank the Health Informatics Centre, University of Dundee for managing and supplying the anonymised data and the Information Statistics Division, NHS Scotland for the supply of cancer registry information. The study was funded from a Breast Cancer Research (Scotland) research grant and a Medical Research Training Fellowship held by $\mathrm{CMcC}$.
Clark TG, Bradburn MJ, Love SB, Altman DG (2003) Survival analysis part I: basic concepts and first analyses. Brit J Cancer 89: $232-238$

Clarke M, Collins R, Darby S, Davies C, Elphinstone P, Evans E, Godwin J, Gray R, Hicks C, James S, MacKinnon E, McGale P, McHugh T, Peto R, Taylor C, Wang Y, Early Breast Cancer Trialists' Collaborative G (2005) Effects of radiotherapy and of differences in the extent of surgery for early breast cancer on local recurrence and 15 -year survival: an overview of the randomised trials. Lancet 366: 2087-2106

Demissie S, Silliman RA, Lash TL (2001) Adjuvant tamoxifen: predictors of use, side effects, and discontinuation in older women. J Clin Oncol 19: $322-328$ 
Dewar JA, Stewart HJ, MacDonald TM (1996) Tamoxifen compliance in a clinical trial. Breast 5: $152-153$

Deyo RA, Cherkin DC, Ciol MA (1992) Adapting a clinical comorbidity index for use with ICD-9-CM administrative databases. J Clin Epidemiol 45: $613-619$

DiMatteo MR (2004) Variations in patients' adherence to medical recommendations: a quantitative review of 50 years of research. Med Care 42: 200-209

Early Breast Cancer Trialists' Collaborative Group (2005) Effects of chemotherapy and hormonal therapy for early breast cancer on recurrence and 15-year survival: an overview of the randomised trials. Lancet 365: $1687-1717$

Early Breast Cancer Trialists' Collaborative Group (2004) Tamoxifen for early breast cancer. Cochrane Database of Systematic Reviews, Reviews 2008 Issue 4. John Wiley \& Sons, Ltd.: Chichester, UK

Fink AK, Gurwitz J, Rakowski W, Guadagnoli E, Silliman RA, Fink AK, Gurwitz J, Rakowski W, Guadagnoli E, Silliman RA (2004) Patient beliefs and tamoxifen discontinuance in older women with estrogen receptor - positive breast cancer. J Clin Oncol 22: $3309-3315$

Fisher B, Costantino JP, Redmond CK, Fisher ER, Wickerham DL, Cronin WM (1994) Endometrial cancer in tamoxifen-treated breast cancer patients: findings from the National Surgical Adjuvant Breast and Bowel Project (NSABP) B-14. J Natl Cancer Inst 86: 527-537

Fisher B, Dignam J, Bryant J, DeCillis A, Wickerham DL, Wolmark N, Costantino J, Redmond C, Fisher ER, Bowman DM, Deschenes L, Dimitrov NV, Margolese RG, Robidoux A, Shibata H, Terz J, Paterson AH, Feldman MI, Farrar W, Evans J, Lickley HL (1996) Five versus more than five years of tamoxifen therapy for breast cancer patients with negative lymph nodes and estrogen receptor-positive tumors. $J$ Natl Cancer Inst 88: 1529-1542

Fisher B, Dignam J, Bryant J, Wolmark N (2001) Five versus more than five years of tamoxifen for lymph node-negative breast cancer: updated findings from the National Surgical Adjuvant Breast and Bowel Project B-14 randomized trial. J Natl Cancer Inst 93: 684-690

Grunfeld EA, Hunter MS, Sikka P, Mittal S, Grunfeld EA, Hunter MS, Sikka P, Mittal S (2005) Adherence beliefs among breast cancer patients taking tamoxifen. Patient Educ Couns 59: 97-102
McDonald HP, Garg AX, Haynes RB (2002) Interventions to enhance patient adherence to medication prescriptions: scientific review. JAMA 288: $2868-2879$

Murthy V, Bharia G, Sarin R (2002) Tamoxifen non-compliance: does it matter? Lancet Oncol 3: 654

Osborne CK (1998) Tamoxifen in the treatment of breast cancer. New Engl J Med 339: $1609-1618$

Osterberg L, Blaschke T (2005) Adherence to medication. N Engl J Med 353: 487-497

Owusu C, Buist DSM, Field TS, Lash TL, Thwin SS, Geiger AM, Quinn VP, Frost F, Prout M, Ulcickas Yood M, Wei F, Silliman RA (2008) Predictors of tamoxifen discontinuation among older women with estrogen receptor positive breast cancer. J Clin Oncol 26: 3125-3127

Partridge AH, Wang PS, Winer EP, Avorn J (2003) Nonadherence to adjuvant tamoxifen therapy in women with primary breast cancer. J Clin Oncol 21: 602-606

Stewart HJ, Forrest AP, Everington D, McDonald CC, Dewar JA, Hawkins RA, Prescott RJ, George WD (1996) Randomised comparison of 5 years of adjuvant tamoxifen with continuous therapy for operable breast cancer. The Scottish Cancer Trials Breast Group. Brit J Cancer 74: 297-299

Sundararajan V, Henderson T, Perry C, Muggivan A, Quan H, Ghali WA (2004) New ICD-10 version of the Charlson comorbidity index predicted in-hospital mortality. J Clin Epidemiol 57: 1288-1294

Swedish Breast Cancer Cooperative Group (1996) Randomized trial of two versus five years of adjuvant tamoxifen for postmenopausal early stage breast cancer. Swedish Breast Cancer Cooperative Group. J Natl Cancer Inst 88: $1543-1549$

Wang J, Donnan PT (2001) Propensity score methods in drug safety studies: practice, strengths and limitations. Pharmacoepidemiology \& Drug Saftey 10: $341-344$

Wei L, Wang J, Thompson P, Wong S, Struthers AD, MacDonald TM (2002) Adherence to statin treatment and readmission of patients after myocardial infarction: a six year follow up study. Heart (British Cardiac Society) 88: $229-233$

Yood MU, Owusu C, Buist DS, Geiger AM, Field TS, Thwin SS, Lash TL, Prout MN, Wei F, Quinn VP, Frost FJ, Silliman RA (2008) Mortality impact of less-than-standard therapy in older breast cancer patients. $J$ Am Coll Surg 206: $66-75$ 